\title{
Neues von der Palmengarten-Gesellschaft 2011 und Rückblick auf das Jahr 2010
}

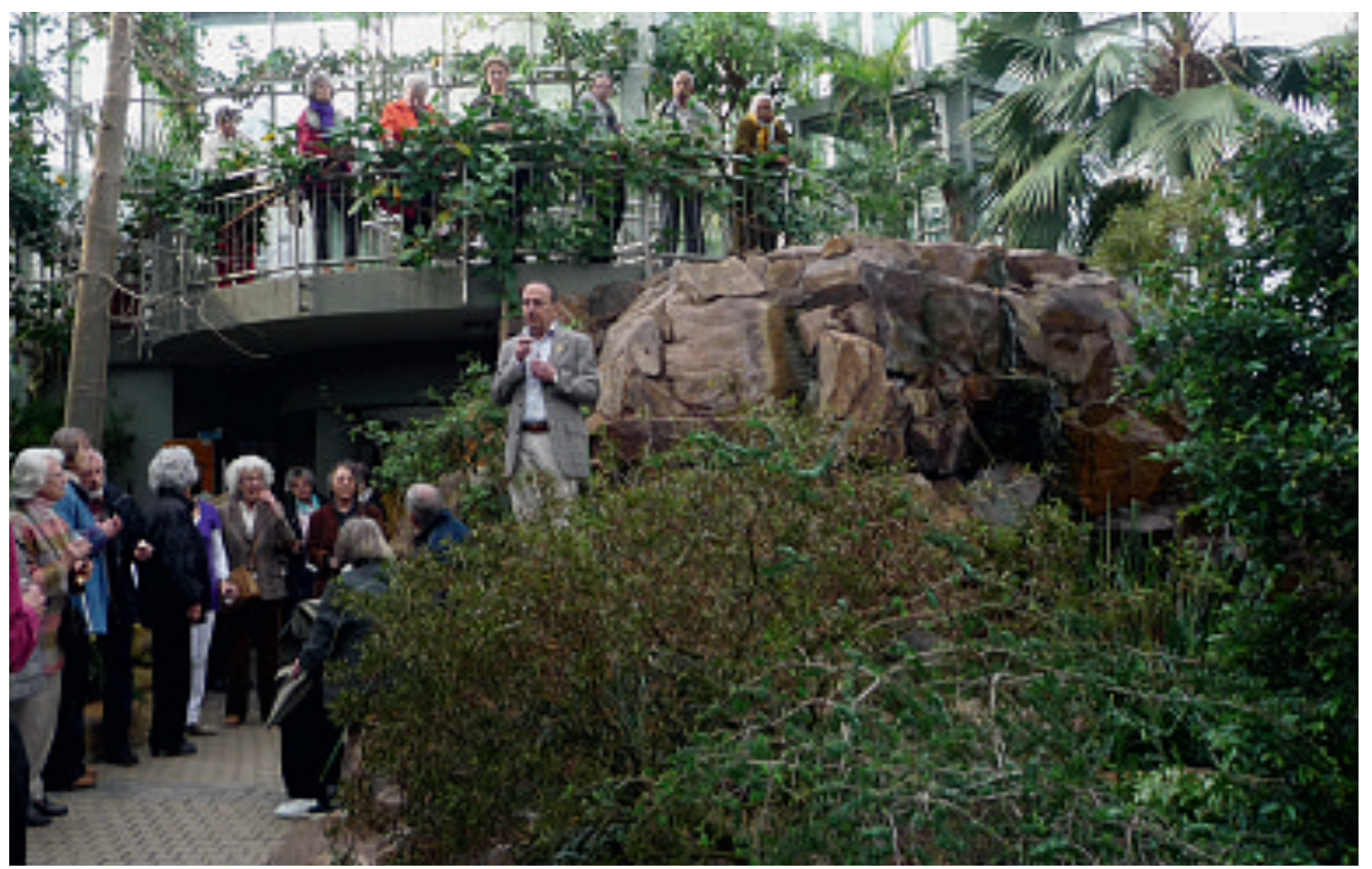

Die Gesellschaft „Freunde des Palmengartens e. V.“ berichtet über ein weiteres erfolgreiches Jahr. 2010 konnte der Palmengarten mit der stolzen Summe von $90000 €(2009: 77000 €)$ gefördert werden. Finanziert wurden davon u.a. Vorträge, Führungen, Schülerseminare, Druck des Palmengarten-Kalenders sowie des Frosch-Imageplakates und der VeranstaltungsProgramme des Palmengartens. Zusätzlich zu dieser finanziellen Unterstützung haben über 50 Mitglieder der Gesellschaft freiwillige Aufsicht bei den Palmengarten-Ausstellungen „StadtGrün“ und „Goethe und die Pflanzenwelt" geleistet. Insgesamt kamen dabei rund 800 Stunden zusammen. Die Mitglieder des Helferkreises haben viele Stunden investiert, um während der Ausstellungen und auf ande-

Abb. 1: Einweihung des Wasserfalls im Trockenwald-Haus. ren Veranstaltungen neue Mitglieder für die Gesellschaft und direkt für den Palmengarten zu werben. Dabei wurden auch die beliebten Palmengarten-Jahressammeltassen und sonstige Produkte für den Palmengarten verkauft. Herzlichen Dank dafür.

Die Zahl der Mitglieder stieg weiter leicht auf nunmehr rund 2400 an. Die Gesellschaft wird zunehmend auch für Jüngere attraktiv. Von den 2011 neu eingetretenen Mitgliedern ist ein Drittel unter 50 Jahre alt.

Jutta Hönig ist seit August 2010 neue Mitarbeiterin in der Geschäftsstelle.

Über die Gesellschaft wurden Tagesfahrten unter Leitung von Dr. Astrid JACOBS nach Bad Nauheim zur Landesgartenschau (48 Teilnehmende) und nach Schlüchtern zum Schloss Ramholz (50 Teilnehmende) angeboten. Die Mehrtagesfahrten führten nach Madeira (17 Teilnehmende) und zum Bodensee zur Insel Mainau (35 Teilnehmende). 


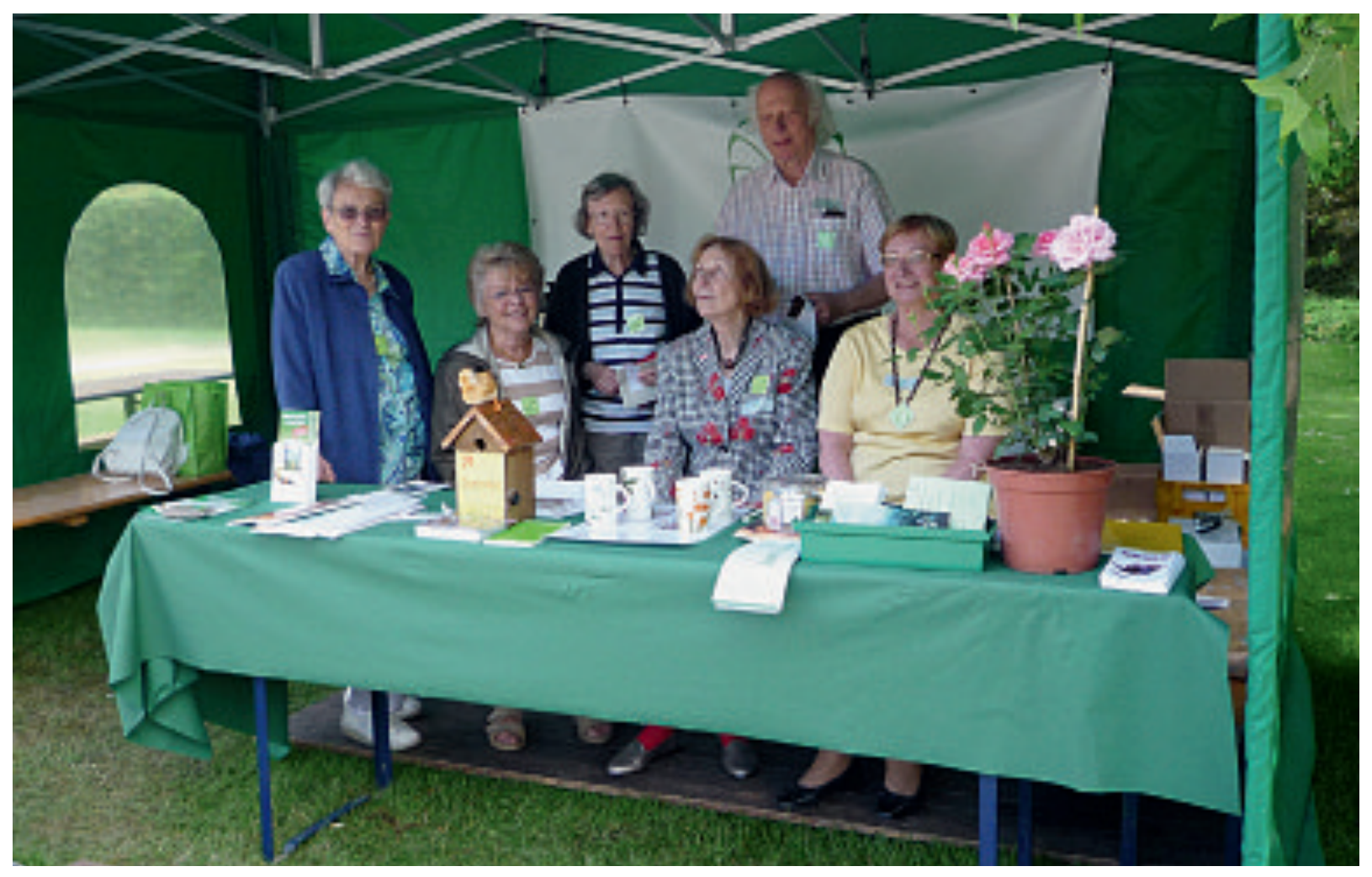

Am 13. Juni 2010, dem letzten Tag der Rosen-Ausstellung, fand ein Sommertreffen für die Mitglieder statt. Organisiert vom Palmengarten war ein musikalischer Frühschoppen am Oktogonbrunnen und ein Promenadenkonzert im Musikpavillon. Auf der großen Wiese vor dem Haus Leonhardsbrunn bestand Gelegenheit zu einem geselligen Gedankenaustausch. Die Kinder konnten am Programm der Grünen Schule teilnehmen. Die Beteiligung war rege.

Das Weihnachtstreffen 2010 fand am 26. November in der Wandelhalle der KfWBankengruppe unter Teilnahme von 168 Mitgliedern statt.

Kamilla Nuyken, langjährige Kassenprüferin der Gesellschaft und große Freundin des Palmengartens, vererbte der Gesellschaft $16000 €$. Auf Vorschlag des Vorstands wird der Palmengarten zum Gedenken an sie einen Baum pflanzen, und zwar eine Hänge-Ulme (Ulmus glabra 'Pendula'). Die im Jahr 2010 durch die Stadt Frankfurt gegründete Stiftung „Palmengarten und Botanischer Garten Frankfurt am Main“ macht es nun auch Mitgliedern der Gesellschaft möglich, Zustiftungen zu leisten. Nähere Auskünfte erteilt die Palmengar- tendirektion oder der Vorstand der Gesellschaft.

In 2011 gab es bereits einige Aktivitäten und wird es noch viele Aktionen geben. So fand die Jahres-Mitgliederversammlung am 13. April 2011 im Großen Hörsaal des Botanischen Instituts statt. Der Mitgliederversammlung unmittelbar voraus ging ein Empfang mit kleinem Umtrunk am neuen Wasserfall am Australien-Beet im Tropicarium. Die Felswand mit Wasserfall wurde von der Gesellschaft mitfinanziert. Während der Jahresmitgliederversammlung wurden langjährige Mitglieder geehrt: Manfred Meyer für 50-jährige, MARgot CASPER für 40-jährige, 9 Mitglieder für 30-jährige und 40 Mitglieder für 25-jährige Mitgliedschaft. Eine neue Bei-

Abb. 2 (S. 74): Fleißige Helferinnen und Helfer werben für die Gesellschaft und den Palmengarten.

Abb. 3 (S. 75, oben): Gemütliches Miteinander von Mitgliedern der Gesellschaft.

Abb. 4 (S. 75, Mitte): Nette Gespräche und Diskussionen mit dem Amtsleiter Matthias Jenny.

Abb. 5 (S. 75, unten): Image-Plakat des Palmengartens. 


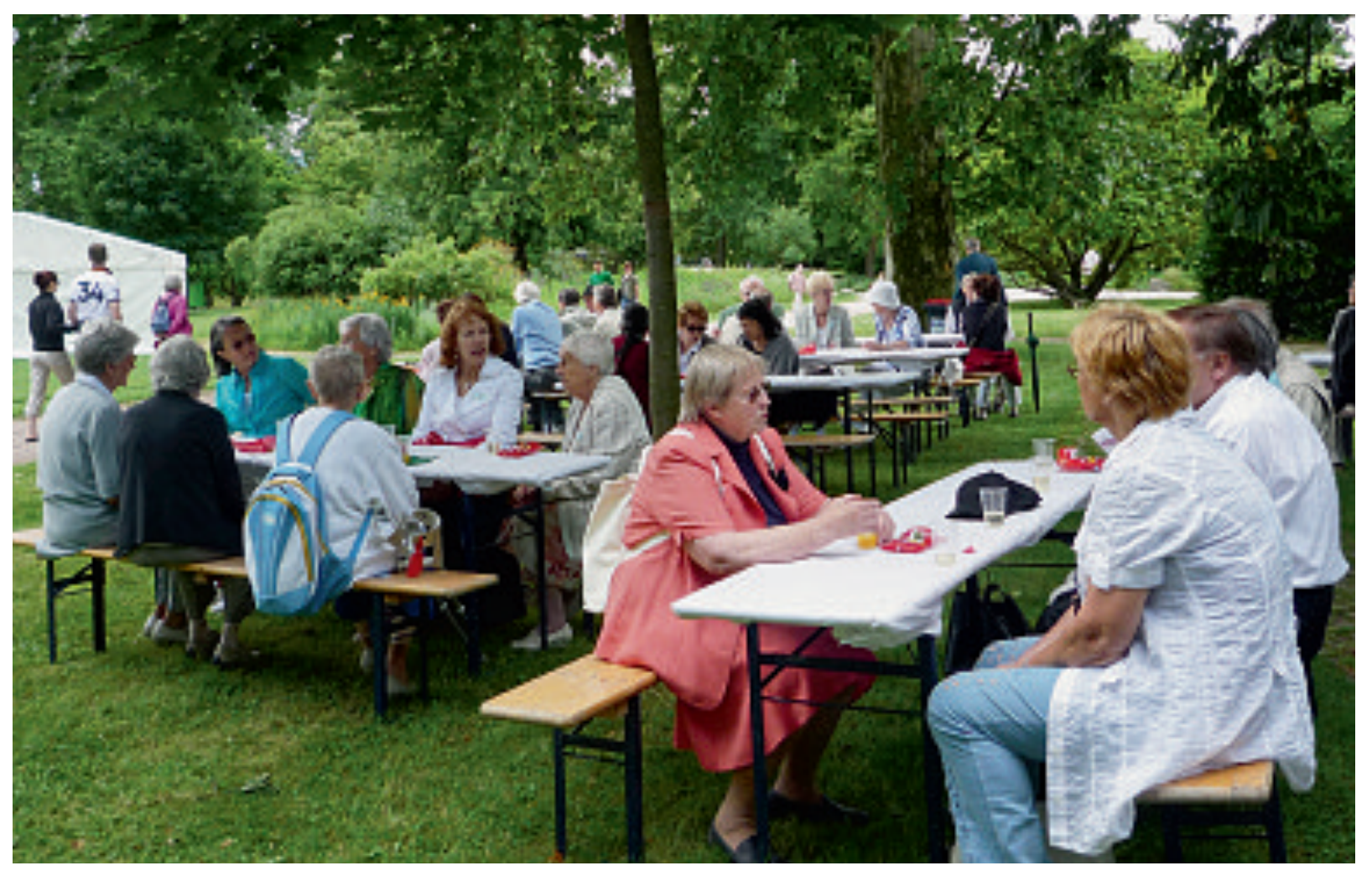

tragsstruktur wurde beschlossen, die ab 2012 gilt und die nachhaltige und direkte Förderung des Palmengartens zum Ziel hat.

Weitere Einzelheiten werden zu einem späteren Zeitpunkt bekannt gegeben.

Für 2011 ist eine Förderung in Höhe von $140000,-€$ eingeplant.

Die erste Mehrtagesreise 2011 hatte Marokko zum Ziel und wurde von 22 Personen gebucht. Auf dem Programm der zweiten Mehrtagesreise 2011 standen Niederösterreich und Wien. Die beiden Eintagesfahrten führten 2011 zur Bundesgartenschau nach Koblenz und nach Rauischholzhausen bei Marburg. Anfragen bzw. Anmeldungen zu Fahrten und Reisen richten Sie bitte direkt an die Veranstalter, d. h. für Tagesfahrten an Dr. Astrid Jacobs (Tel. 0176-51223163) und für Mehrtagesreisen an Ruge-Reisen (Tel. 040-42936366). Das Sommertreffen 2011 fand am letzten Tag der Rosenausstellung (Pfingstmontag, 13. Juni) statt. Das Fest stand im Zeichen des 80-jährigen Bestehens der Palmengarten-Gesellschaft.

Ich wünsche allen ein gutes, interessantes und erfolgreiches Palmengarten-Jahr 2011.

Klaus Urban
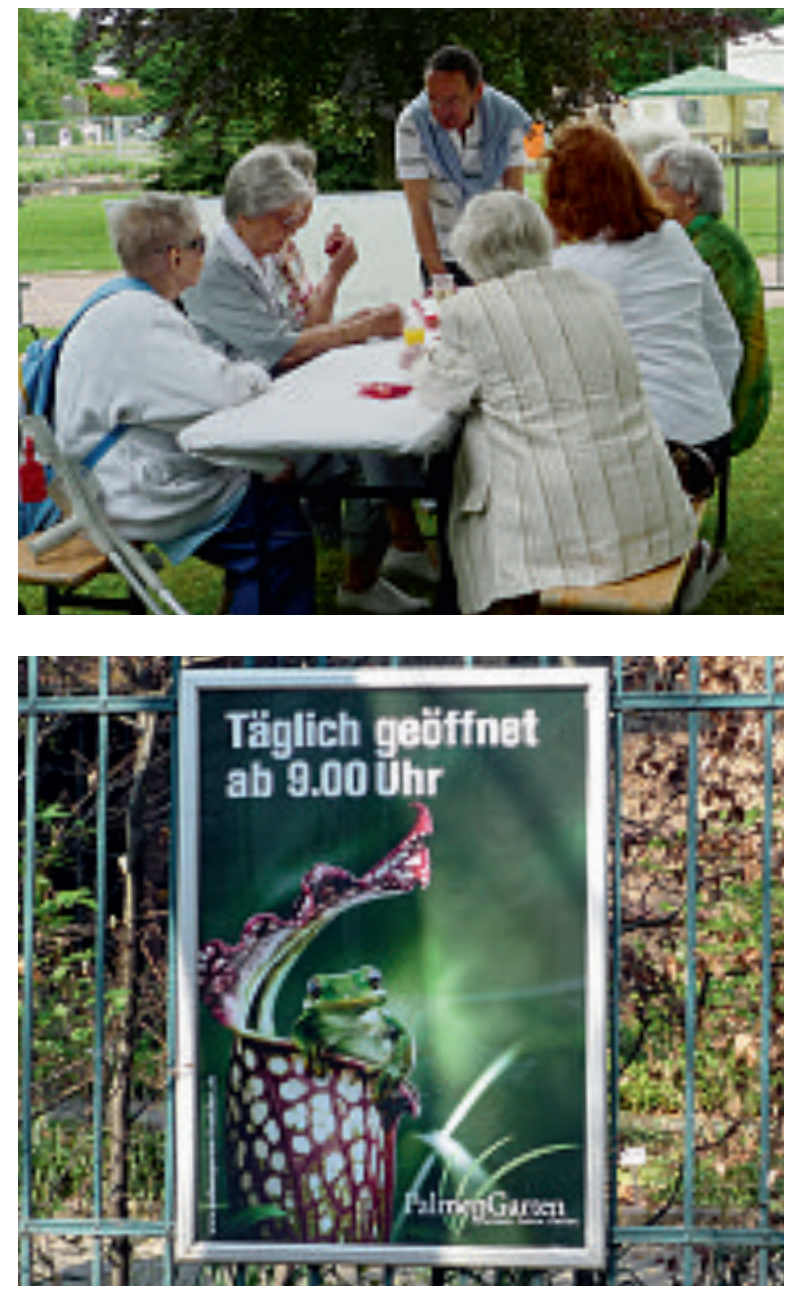